\title{
Response to: Research Integrity: the Experience of a Doubting Thomas
}

\author{
Pere Puigdomènech
}

Published online: 23 February 2014

(c) L. Hirszfeld Institute of Immunology and Experimental Therapy, Wroclaw, Poland 2014

\section{Dear Editor,}

Dealing with cases of scientific misconduct is essential for the scientists because, as Dr. Hettinger says, "it is destructive to science culture". It is therefore important that scientists and scientific institutions deal with these cases when they appear. The case that Dr. Hettinger comments has been very complex for the CSIC's Ethics Committee for several reasons. The case came to attention following a letter of the Editor of Science just a few weeks after the appointment of the Committee and even before its rules of procedure were approved. The article involves 18 authors, two of them corresponding authors, from at least five different institutions and four different countries. It was a complex multidisciplinary article with data from organic chemistry to microbiology. As soon as the note of concern on the article appeared in Science, letters arrived to the Committee including one by Prof. Richard Rogers, Nobel Prize winner that strongly defended the results.

The Committee appointed an ad hoc committee with experts from outside of CSIC that analyzed the article and reached conclusions very similar to those indicated by Dr. Hettinger. The authors of the article and the other institutions involved were asked to comment on the conclusions that were the basis of the recommendation to retract the article. All these actions were carried out within the mandate of the CSIC's Ethics Committee that is an advisory board to the President of CSIC. Any further actions such as the identification of individual responsibilities or to apply disciplinary measures were outside the mandate of the Committee that recommended the President of CSIC to act in this direction.

Science in Spain, as in any other country, works within the established legal framework that varies very much from one country to another. In Spain, CSIC is an institution of the Public Administration and its scientists have the status of civil servants. This situation does not favor any investigation of misconduct as, for instance, scientific fraud is not considered in the statute of civil servants. This limitation has been openly criticized in Spanish journals. Copies of these publications, of course written in Spanish or Catalan, may be made available to the interested reader.

Following the "reactome" case, the CSIC's Ethics committee published a statement of guidance for authors of multidisciplinary articles. In his resolution, the Committee also stated that peer review did only a partial analysis of the results of the article and this opinion was also communicated to the editor of Science. In any case, the experience of the CSIC's Ethics Committee demonstrates the importance and difficulty of dealing with complex cases of alleged misconduct. Those taking on these responsibilities, especially in its initial phases, should be aware that their work will rarely be appreciated by the people involved in the cases they analyze and by people from a distance that have only a partial knowledge of the whole situation.
P. Puigdomènech $(\square)$

Centre for Research in Agricultural Genomics,

CSIC-IRTA-UAB-UB, Edifici CRAG, Campus UAB,

08193 Bellaterra (Barcelona), Spain

e-mail: pere.puigdomenech@cragenomica.es 